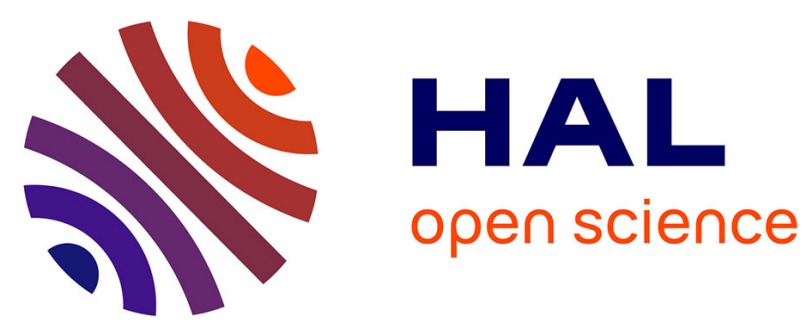

\title{
In-situ layerwise monitoring of electron beam powder bed fusion using near-infrared imaging
}

Guillaume Croset, Guilhem Martin, Charles Josserond, Pierre Lhuissier, Jean-Jacques Blandin, Rémy Dendievel

\section{To cite this version:}

Guillaume Croset, Guilhem Martin, Charles Josserond, Pierre Lhuissier, Jean-Jacques Blandin, et al.. In-situ layerwise monitoring of electron beam powder bed fusion using near-infrared imaging. Additive Manufacturing, 2021, 38, pp.101767. 10.1016/j.addma.2020.101767 . hal-03427100

\author{
HAL Id: hal-03427100 \\ https://hal.science/hal-03427100
}

Submitted on 17 Nov 2021

HAL is a multi-disciplinary open access archive for the deposit and dissemination of scientific research documents, whether they are published or not. The documents may come from teaching and research institutions in France or abroad, or from public or private research centers.
L'archive ouverte pluridisciplinaire HAL, est destinée au dépôt et à la diffusion de documents scientifiques de niveau recherche, publiés ou non, émanant des établissements d'enseignement et de recherche français ou étrangers, des laboratoires publics ou privés. 


\title{
In-situ layerwise monitoring of electron beam powder bed fusion using near- infrared imaging
}

\author{
Guillaume Croset ${ }^{1 \#}$, Guilhem Martin ${ }^{1 \#}$, Charles Josserond ${ }^{1}$, Pierre Lhuissier ${ }^{1}$, Jean-Jacques \\ Blandin ${ }^{1}$, Rémy Dendievel ${ }^{1}$
}

1. Univ. Grenoble Alpes, CNRS, Grenoble INP, SIMaP, F-38000 Grenoble, France

Laboratoire de Science et Ingénierie des Matériaux et Procédés (SIMaP)

Groupe Génie Physique et Mécanique des Matériaux (GPM2)

1130 Rue de la Piscine

38402 Saint-Martin-d'Hères

France

\# Corresponding authors: guillaume.croset@grenoble-inp.org

guilhem.martin@simap.grenoble-inp.fr

DOI: 10.1016/j.addma.2020.101767

\begin{abstract}
:
A near-infrared camera (NIR) is employed to monitor layer-by-layer in-situ electron beam powder bed fusion (E-PBF). We aim at demonstrating that such a device allows various kinds of flaws to be reliably captured thanks to qualitative thermal information. Defects such as distortions or uneven top surfaces can be detected in the NIR-images. Information about temperature heterogeneities can also be linked with some distortions occurring near overhangs. Some lack-of-fusion pores are revealed in the NIR-images. Unexpected localized defects appearing as isolated pores elongated along the building direction, and referred to as chimney pores, are also detected in the NIR-images. Pore-like defects can be described in 3D based on the NIR-images. Post-processing non-destructive characterization performed using Xray computed tomography (XCT) is carried out to validate the information provided by the NIRimages. Comparisons between the 3D images obtained from the NIR-images acquired in-situ and processed using image analysis, and the 3D images obtained through XCT demonstrate that the morphology and spatial distribution of the defects can be reliably captured by NIR-imaging.

Keywords: E-PBF; Near-infrared imaging (NIR); In-situ; Defects; X-ray computed tomography $\underline{(\mathrm{XCT})}$
\end{abstract}




\section{Introduction}

Electron beam powder bed fusion (E-PBF) is an additive manufacturing process that may generate various defects in the built parts. Those defects often lead to a significant knock-down of the mechanical properties, see e.g. [1,2]. First, some defects are directly linked to improper energy input. An insufficient energy input induces lack-of-fusion defects, i.e. porosity with an irregular morphology [1]. On the contrary, an excess of energy leads to geometrical distortions as well as to top surface defects [3]. Other defects are not necessarily linked with energy input but can be attributed to different causes. For example, geometrical distortions can be observed because of missing supports underneath the parts to be fabricated, flaws might appear due to unsuitable machine settings such as a defocused beam or an improper powder supply.

Defect generation reduces the reliability of E-PBF and slows down its development and industrialization. Thus, in-situ imaging of E-PBF is a requirement to detect the presence of different defects as well as their spatial distribution within the parts during the process [4-6]. This information is a relatively efficient way to identify defective parts that would eventually require post-processing treatments such as machining or Hot Isostatic Pressing (HIP) to heal those defects. It becomes also possible to locate the regions with defects, and thus to adjust processing parameters in the next builds. Besides, in the case where in-situ monitoring reveals a particularly harmful defect in parts, then the process could be interrupted to save time and raw materials. Finally, in-situ monitoring is the first step towards a closed-loop control of the process where insitu data would be used to modify processing parameters to avoid defects or to heal them when processing the next layers.

Two main imaging techniques have been investigated for in-situ monitoring of E-PBF in the literature in order to observe the building surface: infrared and electronic imaging. Electronic imaging takes advantage of the interactions between the incident electron beam and the material to be processed to collect secondary and/or back-scattered electrons (BSE) thanks to a dedicated detector introduced in the building chamber. It produces in-situ electronic images of the built parts, similarly as done with an SEM. Such detectors can be positioned near the bottom of the electron gun as reported by Arnold et al. [7], or implemented on heat shields (metallic panels 
designed to limit radiation heat losses) as suggested by Wong et al. [8]. The ability of in-situ electronic imaging to provide information about part geometry, surface topology, and typical defects such as pores or uneven surfaces was demonstrated, see in particular Pobel et al. [9] and Wong et al. [8]. However, up to now, it turns out to be difficult to infer thermal information of the layer to be processed from electronic images.

In-situ infrared imaging is another monitoring technique that has been implemented to improve our current understanding of E-PBF. Various types of cameras were installed: near-infrared (NIR) cameras with a wavelength range generally between $0.7 \mu \mathrm{m}$ and $1 \mu \mathrm{m}$, or mid-infrared (MIR) cameras with working wavelengths longer than $3 \mu \mathrm{m}$. Those instruments allow thermal information to be obtained because a substantial part of the infrared intensity measured by the camera comes from the thermal radiation of the building surface which is directly linked to its temperature and emissivity by the Stefan-Boltzmann law. This information is used for defect detection based on temperature heterogeneities or emissivity variations. The remaining part of the measured infrared intensity comes from radiations emitted by the building chamber environment and reflected by the building surface towards the camera. For example, a part of the radiations emitted by the $\mathrm{W}$-filament (tungsten filament from which electrons are extracted) is reflected by the printed parts. Price et al. [10] used a NIR camera to estimate the temperature of the building surface and reported the thermal effect (slow cooling) linked with the presence of overhang geometries which are thought to be responsible for the limited heat dissipation causing geometrical defects. Boone et al. [11] also employed a NIR camera to establish correlations between the occurrence of "apparent hot spots" in the images, and some defects detected in the final parts such as porosity or swelling. A new strategy to detect hot spots was recently suggested by Grasso et al. [12]. Yoder et al. [13] collected in-situ NIR data about porosity and linked them to the mechanical performances of the built specimens.

Schwerdtfeger et al. [14] used a MIR camera for large pores detection (> $830 \mu \mathrm{m})$ which were, indeed, observed in final parts by optical microscopy. Dinwiddie et al. [15] and Cordero et al. [16] detected pores with a MIR camera as well. Rodriguez et al. [17] calibrated a MIR camera to be able to get an approximation of the absolute temperature of the building surface to improve the thermal monitoring of the process. Raplee et al. [18] also performed a temperature calibration to 
estimate the solid/liquid interface velocity and the thermal gradient, and further linked them to changes in the microstructure of the parts. These few examples demonstrate that key information regarding the defects can be extracted with the help of NIR or MIR cameras. However, reliable measurements of absolute temperatures turn out to be a major difficulty, most likely because the metal emissivity is low $\left(0.37\right.$ for Inconel 718 as-printed surface at $800^{\circ} \mathrm{C}$ and 0.33 for Ti-6Al-4V asprinted surface at $800^{\circ} \mathrm{C}$ for a MIR camera [19]). Furthermore, the emissivity depends on the temperature but also on the nature of the substrate: dense material or dispersed (powder particles) as already reported by several authors $[11,18,20]$. Obtaining reliable temperature measurements requires complex calibration steps for each material $[17,18]$. However, thermal imaging can still provide qualitative thermal information about the building surface. Some NIR cameras allow a higher spatial discretization to be achieved on the complete building surface than MIR cameras, which often exhibit sensors with a lower number of pixels. Thus, NIR cameras are a good compromise to capture a thermal signal while preserving a reasonable spatial discretization.

Here, a high spatial discretization NIR camera is implemented in an ARCAM-A1 E-PBF machine. The first objective is to give an overview of the various possibilities offered by a NIR-camera installed on a E-PBF machine for defect detection allowing to confirm the results reported in the existing literature [10-13] but also to further validate the detected E-PBF defects with the help of image analysis routines specifically developed in the framework of this investigation. With our layerwise monitoring (one image per layer of the whole printing area throughout the build), we aim to confirm that typical E-PBF defects such as lack-of-fusion porosity, chimney pores (elongated pore along the building direction), uneven top surfaces, and geometrical distortions can be detected with the same device by taking advantage of the relatively high spatial discretization and the qualitative thermal information provided by the NIR-images. These defects were either deliberately generated or unexpected in the case of chimney pores. The second objective of this paper is to provide new features regarding the defects captured using NIRimages. As the NIR camera is able to detect defects related to melting parameters out of the processing window (unsuitable energy input), it could also be used to speed-up the optimization of the melting parameters to identify quickly the processing window (beam power versus beam 
scanning speed map) for a given material. The latter being particularly useful in the framework of alloy design for AM. Furthermore, image analysis routines allow the evolution of the printed area of the part being built to be monitored. This piece of information can be helpful to detect issues linked with an excessive energy input giving for instance a too high printed area. Finally, NIRimages allow the in-situ detection of some pore-like defects to be validated. 3D NIR-images of lack-of-fusion and chimney pores were obtained based on novel image processing routines specifically developed for this study and compared with a non-destructive 3D post-processing characterization using X-ray computed tomography (XCT). This validation step is important to assess the presence of the various defects detected in the NIR images, their morphology as well as their spatial distribution within the parts. Ultimately, this validation step helps to determine if there is a possible evolution of a defect detected in the NIR-images at a given layer when processing the subsequent layers. Such a validation step is often missing in past investigations, especially for porosity flaws.

\section{Materials and methods}

\subsection{NIR camera implementation}

Before describing technical details regarding camera implementation, constraints related to EPBF have to be reviewed. This allows our set of requirements to be established. First, the building chamber is under vacuum (typically $5.10^{-4}$ mbar at the end of the pumping step and $2.10^{-3} \mathrm{mbar}$ during the build because helium is introduced in the chamber). The powder bed temperature is around $730^{\circ} \mathrm{C}$ for Ti-6Al-4V. Evaporation of some alloying elements can also occur under vacuum [3], in particular for elements having a low vaporization pressure such as Mg or Al. The evaporated materials might then be deposited on the walls of the building chamber. This strongly reduces transmission through viewports. For all these reasons, the camera is placed outside of the building chamber and allows the build under progress to be monitored through a viewport as schematically shown in Fig. 1.a. A high-temperature resistant and low-outgassing Kapton film is placed between the building surface and the viewport to protect it from evaporated material deposition. The Kapton film is unwound at every single layer during raking operations (powder 
deposition) to avoid a decrease of the NIR transmission throughout the build. Note that the rolling of the Kapton film never occurs while acquiring NIR-images. Interactions between the electron beam and materials produce X-rays. Thus, the viewport includes a material such as leaded glass which stops these radiations, but at the same time transmit the NIR signal. An advantage of working with a NIR camera is that the transmission of leaded glass in the near-infrared range is greater than for longer wavelengths as it has been reported by Boone et al. [11]. A home built version of the film feeder including the above-mentioned Kapton film and leaded glass was designed and implemented. This system, shown in Fig. 1.b, is similar to those developed by others in the literature $[12,19]$.

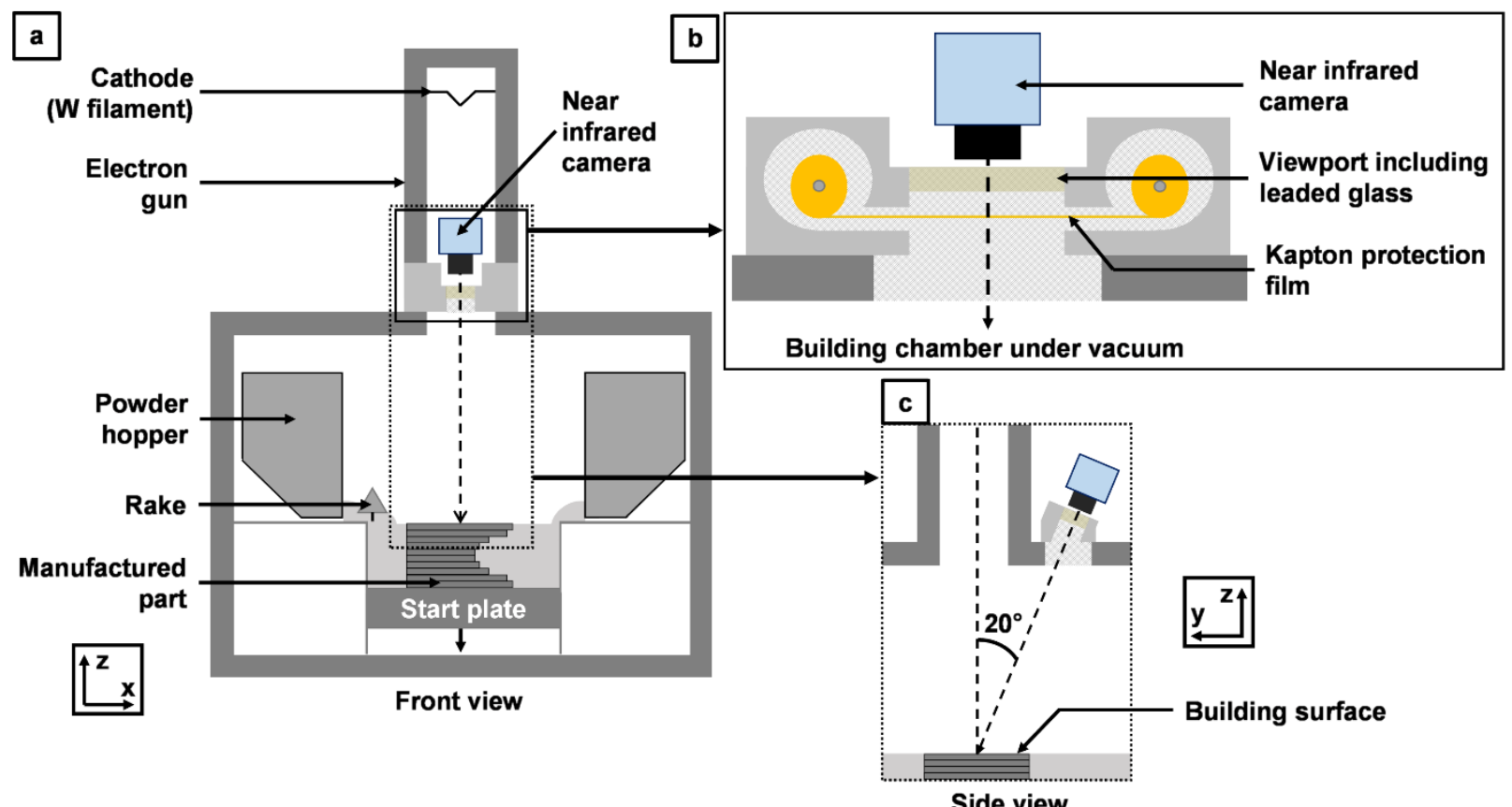

Fig. 1. (a) Schematic front view of the ARCAM-A1 E-PBF machine with the NIR camera implemented. (b) Enlarged schematic view of the home built film feeder system with a viewport including leaded glass for X-ray filtering and the Kapton film for protection against metallization. (c) Side view showing the NIR camera setup and the $20^{\circ}$-angle between the camera optical axis and the normal of the building surface. 
The NIR camera is a Baumer VCXU-123M with a 12 Mpixels CMOS sensor working in the wavelength range from $400 \mathrm{~nm}$ to $1000 \mathrm{~nm}$. It was implemented in an ARCAM-A1 E-PBF machine operating at a constant accelerating voltage of $60 \mathrm{kV}$. To make easier image analysis operations, a $700-1000 \mathrm{~nm}$ band-pass filter was added to the camera because preliminary tests revealed that this wavelength range reduces reflections attributed to the light emitted by the $\mathrm{W}$-filament. Those reflections decrease the efficiency of the image analysis routines developed in this contribution. The camera allows a spatial discretization of approximately $75 \mu \mathrm{m}$ to be obtained over the complete $210 \times 210 \mathrm{~mm}^{2}$ building surface of the ARCAM-A1 E-PBF machine. Image acquisition is controlled automatically by an external computer and is synchronized with information recorded in the ARCAM log studio files. These files contain a lot of information about the building process such as the beam parameters applied for every single melting step (beam current, focus offset which controls the focus of the electron beam), use of the raking system, or

data measured by sensors and recorded in real-time during the build. For example, the temperature measured using a thermocouple inserted in a drilled hole in the build substrate or the pressure within the chamber are recorded throughout the build. In the present work, an image is taken automatically at every single layer once all the parts are selectively melted. The ARCAM log studio files can be readable using a text editor allowing to synchronize image acquisition with information recorded in those ARCAM log studio files. In the present case, image acquisition was synchronized with one of the signals allowing to control the rake for powder deposition. Finally, as there is a $20^{\circ}$-angle between the optical axis of the camera and the normal to the powder bed (see Fig. 1.c), raw images need to be corrected. This will be detailed in the section dedicated to the image analysis routines. Note that the $z$ axis in Fig. 1 corresponds to the building direction.

\subsection{Processing conditions}

To evaluate the ability to detect E-PBF defects with the NIR camera, cuboid samples were manufactured with a powder batch of Ti-6AI-4V ELI (Grade 23) supplied by ARCAM with a particle size between 45 and $100 \mu \mathrm{m}$. Each layer was built with the same processing sequence. First, a 
powder layer was deposited on the building surface. Then, a defocused electron beam scanned the building surface to heat the powder bed to consolidate it. Finally, a focused electron beam selectively melted the parts starting first with contours and then filling in the part interior. The building surface was then lowered by the height corresponding to the layer thickness $(50 \mu \mathrm{m})$ and a new layer of powder was deposited. These steps were repeated until the completion of the build. E-PBF parts were built with a support structure under them. The aim of that support structure was: (i) to allow a proper heat evacuation, (ii) to make easier parts removal from the building substrate, and (iii) to avoid or at least limit geometrical distortions caused by thermal stresses [21,22]. The fabrication of each individual sample was monitored with the NIR-camera.

The fabricated parts are classified as $U$ (uneven), $\mathrm{G}$ (good), or $\mathrm{P}$ (porous) depending on the melting parameters (energy input). This classification is done based on the top surface of the printed parts produced using different melting conditions as shown in Fig 2. Three samples were built with the parameters given in Table 1 and allow the three different categories: $U, G$ and $P$ to be qualitatively illustrated.
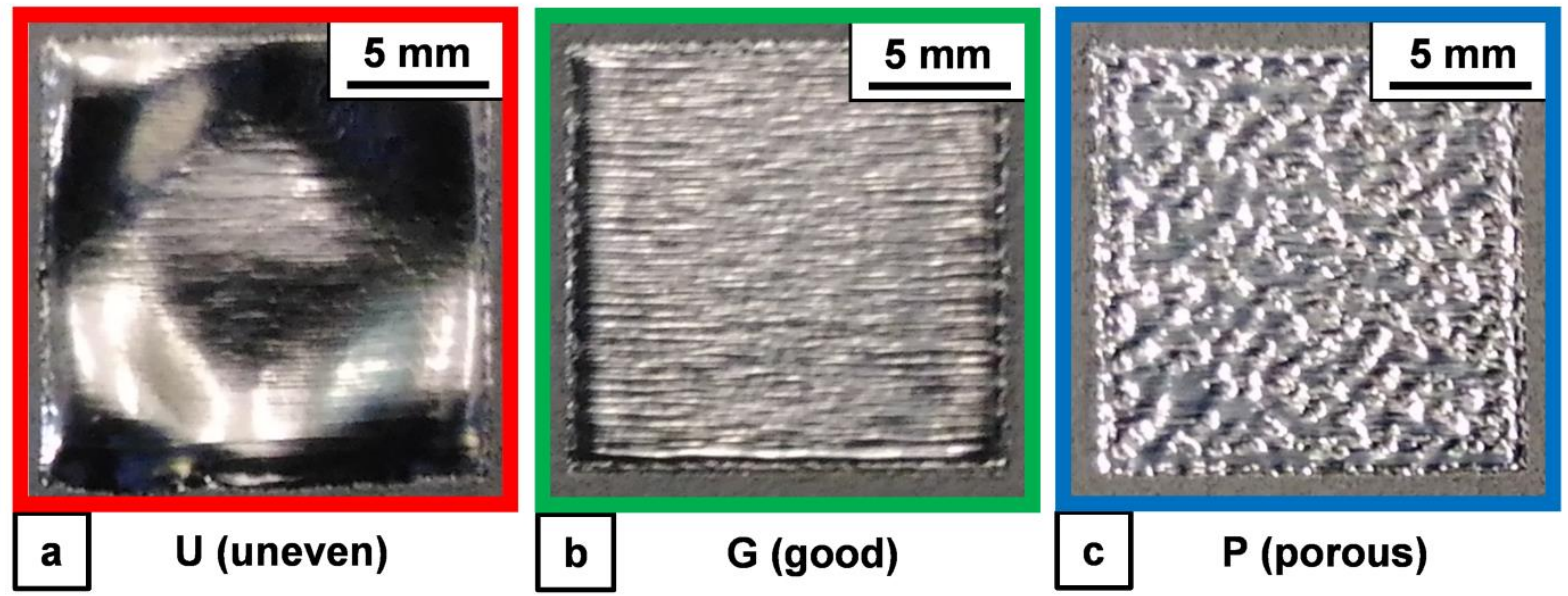

Fig. 2: Images of the top surface of E-PBF samples built with the three typical melting conditions: (a) $U$ (uneven), (b) G (good) and (c) P (porous). Sample size: $15 \times 15 \times 15 \mathrm{~mm}^{3}$. 


\begin{tabular}{|c|c|c|c|}
\hline Sample & U (uneven) & G (good) & $P$ (porous) \\
\hline Sample size $\left(\mathrm{mm}^{3}\right)$ & $15 \times 15 \times 15$ & $15 \times 15 \times 15$ & $15 \times 15 \times 15$ \\
\hline Height of supports (mm) & 5 & 5 & 5 \\
\hline Accelerating voltage (kV) & 60 & 60 & 60 \\
\hline Hatching beam current (mA) & $4.5^{*}$ & $4.5^{*}$ & $4.5^{*}$ \\
\hline Hatching beam power $p(W)$ & 270 & 270 & 270 \\
\hline Hatching strategy & Snake & Snake & Snake \\
\hline Hatching line offset (mm) & 0.1 & 0.1 & 0.1 \\
\hline Hatching focus offset (mA) & 3 & 3 & 3 \\
\hline Hatching speed function & 18 & 98 & 300 \\
\hline Hatching scanning speed $v(\mathrm{~mm} / \mathrm{s})$ & $157^{*}$ & $903 *$ & $2521^{*}$ \\
\hline Line energy $E_{l}(\mathrm{~J} / \mathrm{mm})$ & 1.72 & 0.30 & 0.11 \\
\hline Top surface aspect & $\begin{array}{c}\text { Uneven } \\
\text { (see Fig. 2.a) }\end{array}$ & $\begin{array}{c}\text { Good } \\
\text { (see Fig. 2.b) }\end{array}$ & $\begin{array}{c}\text { Porous } \\
\text { (see Fig. 2.c) }\end{array}$ \\
\hline
\end{tabular}

Table 1. Processing parameters used to produce the samples illustrating the three melting conditions $U, G$ and $P$ (see

Fig.2 for top surface images of these samples). The parameters with an * (hatching beam current and the hatching scanning speed) are not chosen directly by the user in the EBM Control 3.2 software that controls the beam and the melting strategy in the ARCAM-A1 machine. Thus their respective values were calculated from the ARCAM log studio files. The hatching beam current depends on the part geometry, and the scanning speed can be modified using the speed function parameter (the higher the speed function, the higher the scanning speed).

As shown in Table 1, the hatching beam power $\mathrm{p}$ was kept constant and equal to $270 \mathrm{~W}$ for melting the part interior, this corresponds to a beam current of $4.5 \mathrm{~mA}$ given that the accelerating voltage is constant and equal to $60 \mathrm{kV}$. To intentionally generate defects (typically for samples $\mathrm{U}$ and $\mathrm{P}$ ), the hatching scanning speed $\mathrm{v}$, was modified by adjusting the speed function as shown in Table 1 while the focus offset and melting strategy were kept constant. The melting strategy consisted first of melting the contours (beam current of $4 \mathrm{~mA}$ and $10 \mathrm{~mA}$, and spot time of $0.8 \mathrm{~ms}$ and 1.3 ms for respectively the outer and inner contours). Then the part interior was melted with a hatching strategy by moving the beam in a snake-like way with a beam current of $4.5 \mathrm{~mA}$, a 
hatch spacing (line offset) of $0.1 \mathrm{~mm}$ and with varying speed function as detailed in Table 1 . The melting strategy is set so that the hatching direction rotates $90^{\circ}$ between each layer.

Modifying the scanning speed altered the linear energy, $E_{I}=p / v$ supplied by the electron beam. As the beam power $\mathrm{p}$ was kept constant, high scanning speeds led to a decrease of the linear energy. If this energy was too low, lack-of-fusion defects (i.e. pores) were generated (see typically the melting conditions of the $\mathrm{P}$ sample reported in Table 1). On the contrary, slow scanning speeds led to an increase of the linear energy and caused geometrical distortions (melting conditions of the $U$ sample in Table 1) such as uneven top surfaces or deviations to the CAD (Computer Assisted Design) geometry. The good processing conditions correspond to an intermediate energy range which is expected to be within the processing window of Ti-6Al-4V and therefore should not lead to the defects invoked previously.

Table 2 summarizes the processing parameters used to produce the other samples, namely $U 1$, $P 1, G 1$ and G2. Samples U1 and P1 were first built with melting parameters qualified as good before a modification of the speed function to generate respectively geometrical distortions (Urange), and lack-of-fusion pores (P-range). Samples G1 and G2 are used to illustrate the case of defects which are not necessarily linked to unsuitable energy input. Standard melting parameters recommended by the machine supplier ARCAM (G-range) were applied but defects were still observed. Note that the G1-sample melted with the parameters reported in Table 2, was deliberately built with a missing support structure whereas the occurrence of pores in sample G2 was unexpected. 


\begin{tabular}{|c|c|c|c|c|c|c|}
\hline Sample & \multicolumn{2}{|c|}{ U1 } & \multicolumn{2}{|c|}{ P1 } & G1 & G2 \\
\hline Sample size $\left(\mathrm{mm}^{3}\right)$ & $15 \times 15 \times 15$ & $15 \times 15 \times 15$ & $15 \times 15 \times 15$ & $15 \times 15 \times 15$ & $20 \times 20 \times 10$ & $20 \times 20 \times 10$ \\
\hline Height of supports (mm) & 5 & 5 & 5 & 5 & 10 & 10 \\
\hline Building height $\mathrm{z}(\mathrm{mm})$ & $15<z<17.7$ & $17.7<z<20$ & $13<z<16.3$ & $16.3<z<20$ & All & All \\
\hline Accelerating voltage (kV) & \multicolumn{2}{|c|}{60} & \multicolumn{2}{|c|}{60} & 60 & 60 \\
\hline $\begin{array}{l}\text { Hatching beam current } \\
\qquad(\mathrm{mA})\end{array}$ & \multicolumn{2}{|c|}{$4.5^{*}$} & \multicolumn{2}{|c|}{$4.5^{*}$} & $17.4^{*}$ & $17.4^{*}$ \\
\hline $\begin{array}{l}\text { Hatching beam power } p \\
\text { (W) }\end{array}$ & \multicolumn{2}{|c|}{270} & \multicolumn{2}{|c|}{270} & $1044^{*}$ & $1044^{*}$ \\
\hline Hatching strategy & \multicolumn{2}{|c|}{ Snake } & \multicolumn{2}{|c|}{ Snake } & Snake & Snake \\
\hline Hatching line offset (mm) & \multicolumn{2}{|c|}{0.1} & \multicolumn{2}{|c|}{0.1} & 0.1 & 0.1 \\
\hline Hatching focus offset (mA) & \multicolumn{2}{|c|}{3} & \multicolumn{2}{|c|}{3} & 3 & 3 \\
\hline Hatching speed function & 38 & 18 & 158 & 300 & 98 & 98 \\
\hline $\begin{array}{l}\text { Hatching scanning speed v } \\
\qquad(\mathrm{mm} / \mathrm{s})\end{array}$ & $318^{*}$ & $157^{*}$ & $1381^{*}$ & $2521 *$ & $5239 *$ & $5239 *$ \\
\hline Line energy $E_{l}(\mathrm{~J} / \mathrm{mm})$ & 0.85 & 1.72 & 0.20 & 0.11 & 0.20 & 0.20 \\
\hline $\begin{array}{c}\text { Defects observed in NIR- } \\
\text { images }\end{array}$ & No & $\begin{array}{c}\text { Too large } \\
\text { printed area }\end{array}$ & No & $\begin{array}{l}\text { Lack-of- } \\
\text { fusion pores }\end{array}$ & $\begin{array}{c}\text { Overhang } \\
\text { distortion } \\
\text { because of a } \\
\text { missing } \\
\text { support }\end{array}$ & $\begin{array}{l}\text { Chimney- } \\
\text { pores }\end{array}$ \\
\hline
\end{tabular}

Table 2. Summary of processing parameters used to produce samples U1, P1, G1 and G2. The parameters with an * are not chosen directly by the user in the EBM Control 3.2 software that controls the beam and the melting strategy in the ARCAM-A1 machine. Those values were rather extracted from the ARCAM log studio files. The hatching beam current depends on the part geometry, and the scanning speed can be modified using the speed function parameter.

\subsection{Image analysis and post-processing characterization}

Once the build is completed, the first step is to correct the optical distortion of in-situ NIR-images which is caused by the fact that the camera is tilted with respect to the normal of the powder bed (angle of $20^{\circ}$, see Fig. 1.c). This correction is done by applying an image transformation based on the acquisition of a calibration pattern before the start of the build. The calibration pattern consists of 16 control points randomly positioned on a $35 \times 35$ regular grid. It is first placed on top of the start plate, an image is taken with the camera (image A) and compared with the original 
pattern (image B) as it can be seen in Fig. 3. The transformation from image $A$ to image B, made using B-splines, is computed with the bUnwarpJ plugin available in ImageJ, see references $[23,24]$ for more details. This transformation is then systematically applied to all NIR-images acquired insitu to correct the image distortions.
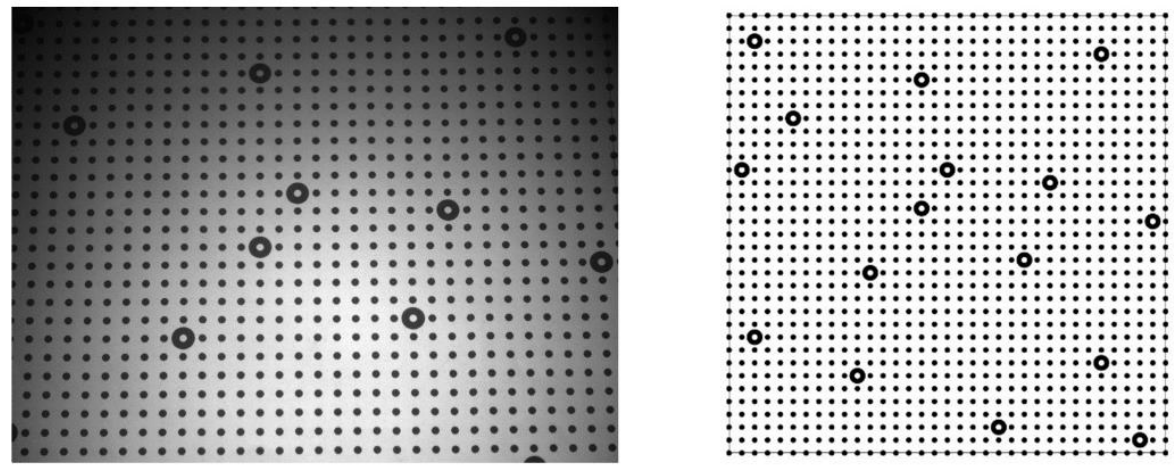

Image A

Calibration pattern on the start plate (taken with the NIR-camera)

$\longrightarrow$ Original calibration pattern

Transformation A $\rightarrow$ B

computed with bUnwarpJ

(ImageJ plugin)

Fig. 3. Procedure used to compute the transformation which is applied to NIR-images to correct image distortions. The image of the calibration pattern on the start plate is taken prior to the build.

For the sample U1, the energy provided by the electron has been modified in order to generate geometrical distortions, an image analysis routine was developed in order to investigate the evolution of the printed area linked to this energy variation based on the NIR-images. The objective of this image analysis routine developed using ImageJ is to detect the boundaries of the parts thanks to the emissivity gradient between the powder bed and the melted material (bulk parts). For a given material at a given temperature, the powder emissivity is higher than the emissivity of the dense material because of the difference of surface topology $[18,20]$. This statement has been experimentally verified for the Ti-6Al-4V alloy by Dinwiddie et al. [19]. This phenomenon induces a grey level gradient at the part boundaries, see e.g. the NIR-image in Fig. 4.a. This grey level gradient was better revealed by plotting a grey level profile through the part 
as shown in Fig. 4.b. The latter could be used to identify part boundaries by following the four steps procedure described below. (i) First, thermal gradients within the powder bed and within the parts can be observed in Fig. 4.b. While the latter may contain useful information about the building process, it becomes an issue when identifying the boundaries. Thus, the first step was to perform a "normalization" of all NIR-images to get rid of these thermal gradients using a background correction. This operation was made by dividing the $\mathrm{i}^{\text {th }}$-image by a Gaussian blurred version of the same $\mathrm{i}^{\text {th }}$-image. It can be seen in Fig. 4.c that, after background correction, thermal gradients are removed whereas the emissivity gradient remains. The obtained image is shown in Fig. 4.d. (ii) A manual cutoff threshold was then applied to obtain binary images of the boundaries thanks to the remaining emissivity gradient. On these binary images, boundaries are white while the rest of the image is black (see in Fig. 4.d). (iii) Subsequently, it can be seen that the inner side of the peaks corresponds to the real boundaries in Fig. 4.c. Thus, the black area which is inside the boundaries in binary images corresponds to the printed surface. Binary images of the boundaries were thus converted to binary images of the printed surface simply by filling the exterior black surface with white and by inverting the image (see in Fig. 4.d). (iv) Finally, as the remaining white area corresponds to the printed surface, it was computed based on the spatial discretization. As the cutoff threshold was kept constant for all 2D-images, it was possible to evaluate the evolution of the printed area of a given part throughout the build. 


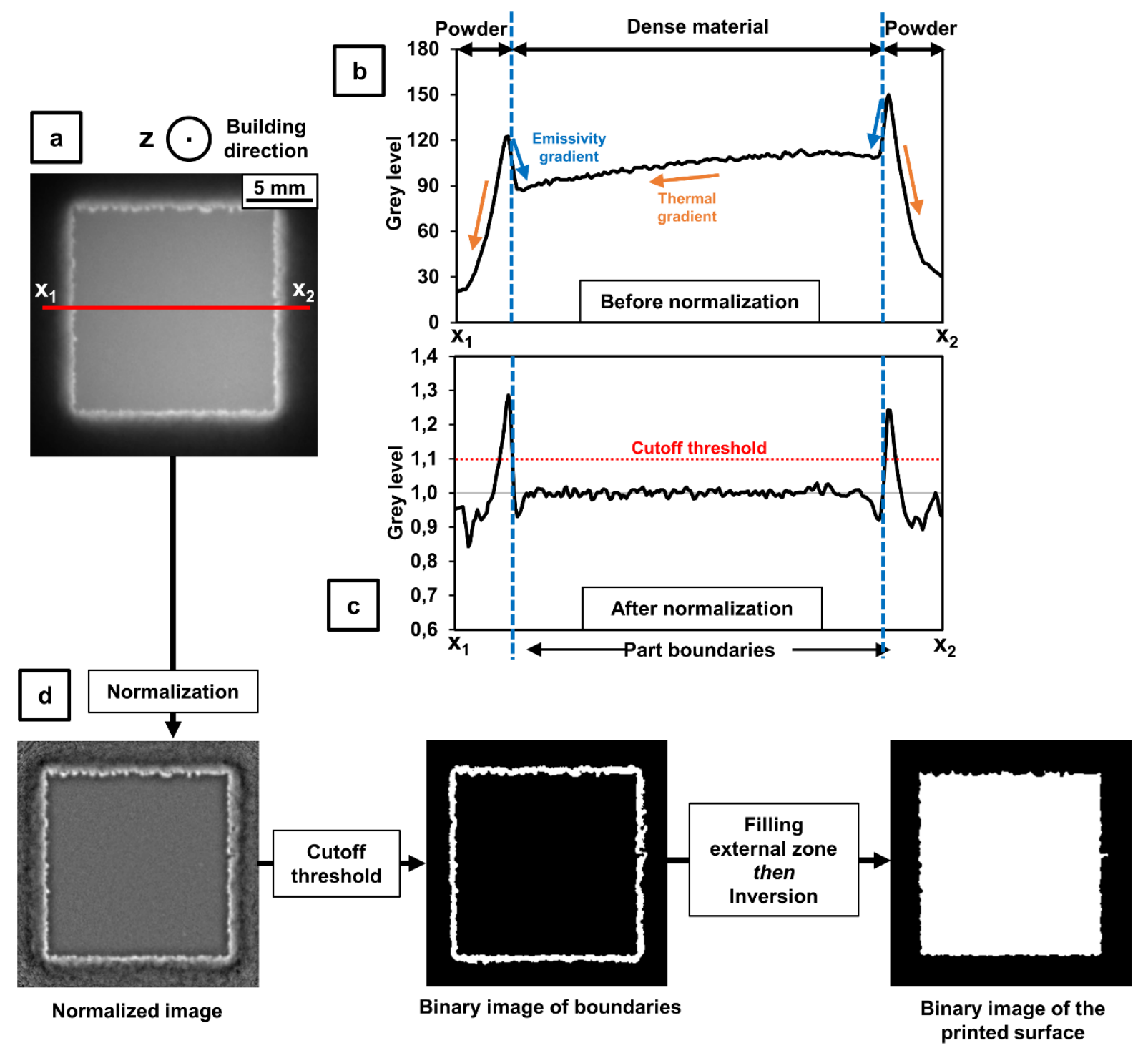

Fig. 4. Illustration of the part boundaries detection image processing routine. (a) NIR-image of a cube for which the grey level is plotted along the line defined by the two points $x_{1}$ and $x_{2}$ in (b). This grey level profile is affected by thermal gradients in the part, in the powder bed but also by the emissivity gradient between powder and dense material. The same grey level profile is plotted in (c) after NIR-image normalization subsequently to a background correction. Note that the background correction allows the thermal gradient to be removed but allows the emissivity gradient to be preserved. A final cutoff threshold can be applied to the corrected images to identify part boundaries. (d) Example showing the image processing steps to obtain a binary image of the printed surface.

To validate the defect detection based on in-situ NIR-images, a comparison with post-processing characterization of the defects is required. While the characterization of geometrical distortions 
or surface aspect is relatively obvious based on visual inspection for the samples U1 and G1 presented in this paper, the porosity detected in samples P1 and G2 requires an additional procedure to be characterized in 3D (see Fig. 5). Here, NIR-images were processed with in-house image analysis routines using ImageJ software to obtain the 3D volume showing pores in the parts with the procedure described below. (i) Similarly to the part boundaries detection described previously, a normalization was applied to all NIR-images to suppress the thermal gradient without affecting pore-like defects. (ii) Then, an identical manual cutoff threshold was applied to all images to obtain binary images of pores. (iii) It was also helpful to apply some complementary operations (such as a 3D median filter) to remove the remaining noise introduced by reflections linked with the tungsten W-filament. (iv) Finally, 2D images are stacked to generate a 3D image of pores contained in parts. The NIR 3D image is then compared with the 3D image resulting from a non-destructive post-processing characterization of the fabricated parts and carried out by Xray computed tomography $(\mathrm{XCT})$. Scans were acquired with a EasyTom $\mathrm{XL}$ tomograph (RXsolution). 1056 projections (exposure time of $1.5 \mathrm{~s}$ ) over $360^{\circ}$ were acquired for each sample using an X-ray incident beam $(150 \mathrm{kV}, 60 \mu \mathrm{A})$ filtered by $0.5 \mathrm{~mm}$ of copper and a geometric magnification of 8.5 which results in a voxel size of $15 \mu \mathrm{m}$. 3D volumes were reconstructed with the Xact software using standard filtered back-projection algorithm. The data were processed with ImageJ: a manual cutoff threshold is applied on background corrected images to identify pores which are often filled with partially-melted powder particles. These particles can be partly suppressed for those which are not in contact with dense material. Some complementary operations allow residual noise to be suppressed. Finally, a 3D image showing pores can be generated and compared with the NIR 3D image. This comparison is crucial to make sure that flaws that were suspected based on the in-situ NIR-imaging correspond to real defects in the final parts. Indeed, one could imagine that a defect appearing at a given layer could be corrected, or, on the contrary, propagated when processing the next layers. It should be noted that the objective was not to establish a quantitative comparison of the results obtained using the two techniques because the spatial discretization difference between the 3D NIR-image (voxel size of $75 \times 75 \times 50 \mu \mathrm{m}^{3}$ ) and the XCT 3D volume $\left(15 \times 15 \times 15 \mu \mathrm{m}^{3}\right.$ voxel size) was too significant. 


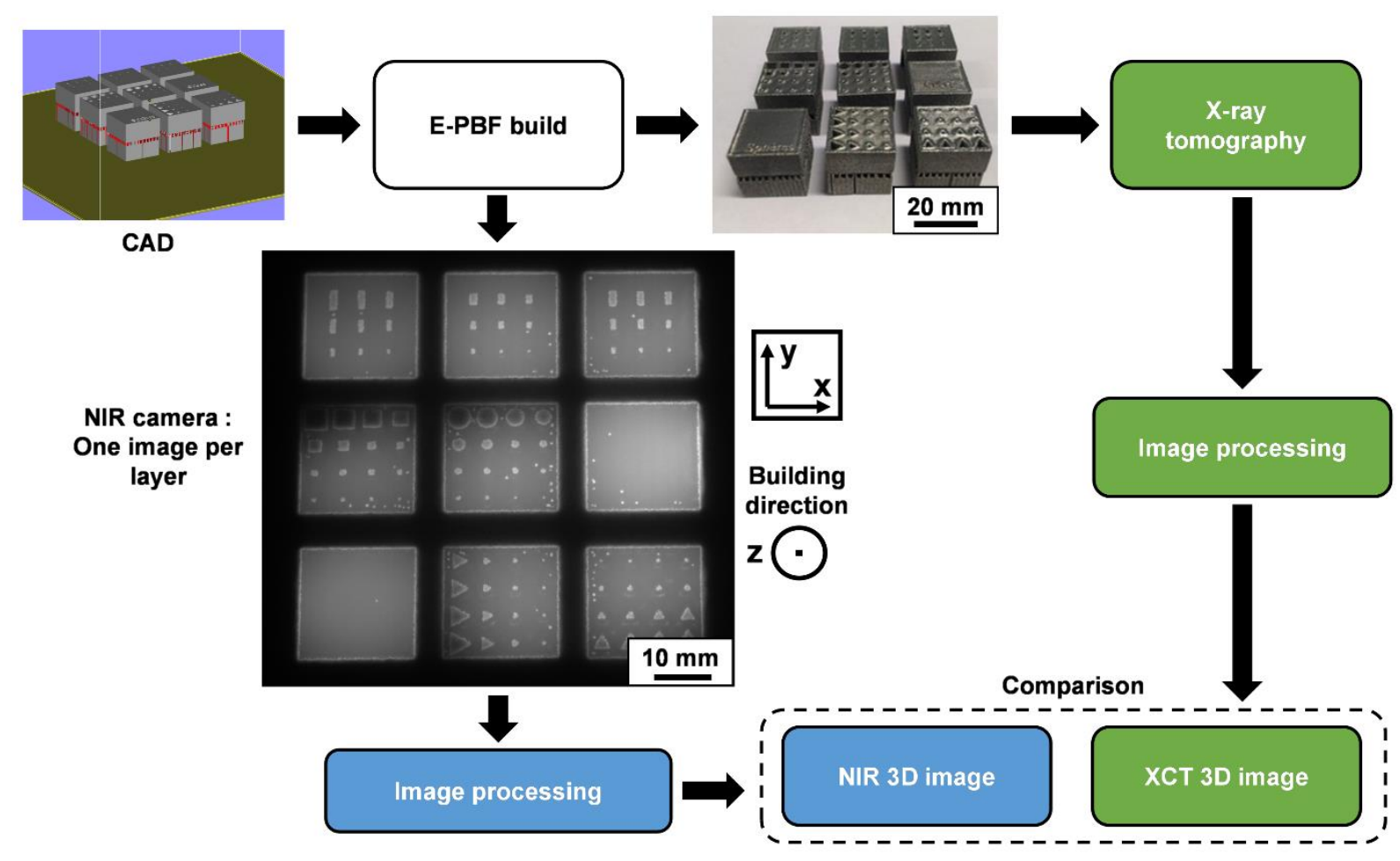

Fig. 5. Summary of the experimental procedure for porosity detection and final validation by XCT. In-situ NIR-images taken during the build are processed to obtain a 3D description of pores. XCT-scans allow a 3D image of the defects in the final parts to be obtained, the latter being compared with the 3D NIR-image. Sample size: $20 \times 20 \times 10 \mathrm{~mm}^{3}$.

\section{In-situ defect detection and post-processing validation}

Fig. 6.a shows a classical schematic plot of beam power as a function of scanning speed where the three different typical regimes can be identified. For the following experiments, beam power was kept constant $(270 \mathrm{~W})$. Depending on the processing parameters, samples with contrasted quality can be produced: $U$ (uneven), G (good), and P (porous). Uneven samples (see the U sample in Table 1), are often associated to an excessive energy input that leads to uneven top surfaces as exemplified in Fig 6.b. Samples qualified as good (G in Table 1) exhibit a relative density higher than 99.8 \% based on XCT-scans and a relatively flat top surface in the optical image shown in Fig 6.c; horizontal stripes corresponding to adjacent molten tracks due to the hatching strategy can be observed. The associated NIR-image shows a part with a uniform grey level. Porous samples ( $P$ in Table 1) result from an insufficient energy input leading to the formation of pores, see Fig 
6.d. This will be further illustrated later in the subsequent sections of the present manuscript. It is worth noting that the NIR camera allowed the processing window of the Ti-6Al-4V alloy with a $270 \mathrm{~W}$ beam power to be quickly determined just by looking to the occurrence of defects associated with each melting condition in the NIR-images. Thus, samples produced with a line energy lower than $0.14 \mathrm{~J} / \mathrm{mm}$ were porous $(P)$ and those produced with a line energy above 1.08 $\mathrm{J} / \mathrm{mm}$ were uneven (U). Thus, as already suggested by Pobel et al. [9] with in-situ electronic images, NIR-images could be helpful to determine the processing window for a new material in a more efficient way. In addition, as it is possible to modify beam parameters during the build, healing of defects during processing the next layers can be studied. 


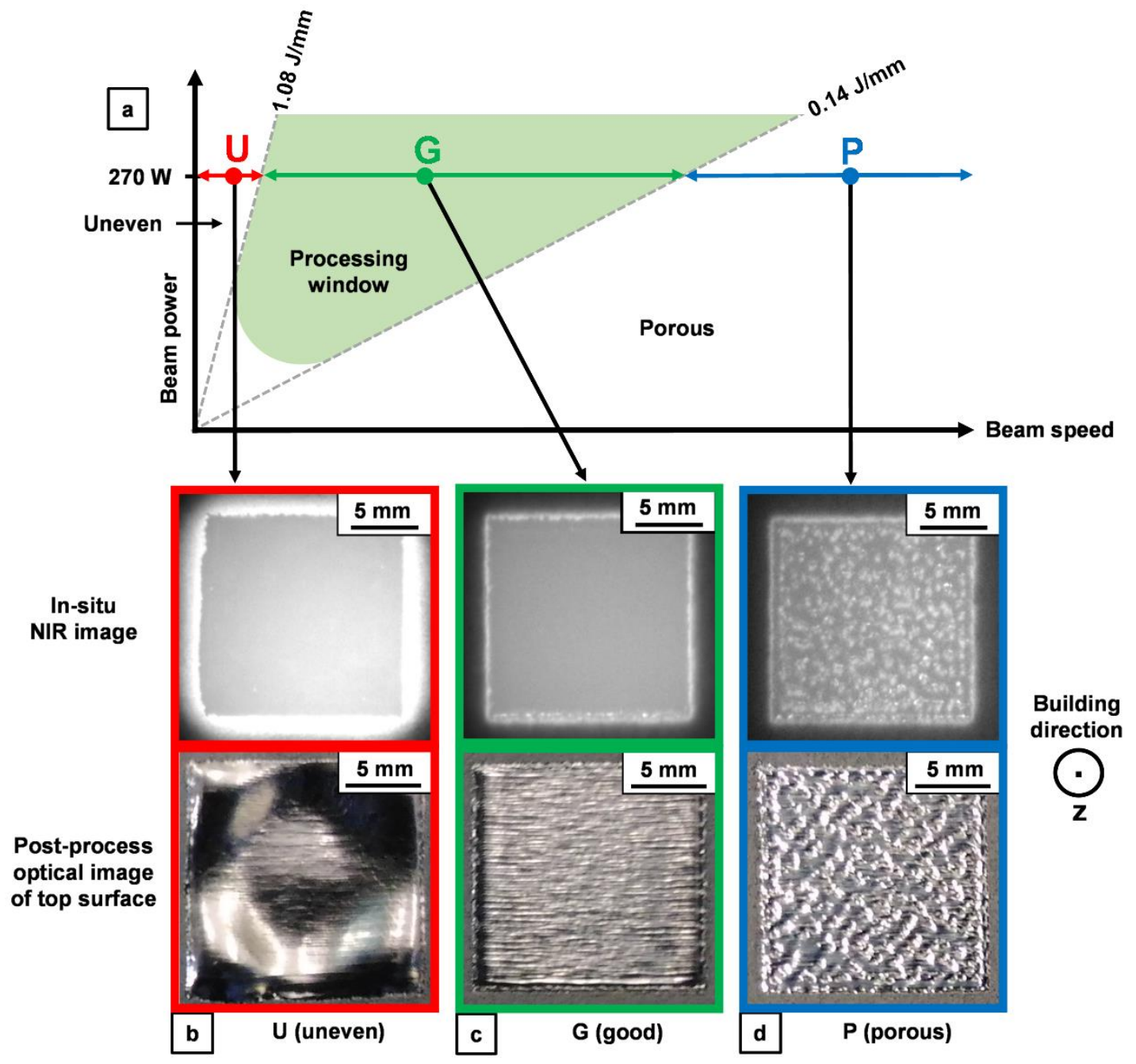

Fig. 6. (a) Schematic beam power-scan speed diagram showing typical processing conditions with associated in-situ NIR-images and top surface post-process observations (optical images) for (b) an excessive energy input giving an uneven top surface, (c) a suitable energy input and (d) a too low energy input giving a porous sample. Note that NIRimages are sub-surface images taken during the build. The upper and lower energy boundaries of the processing window were determined directly by defects observations on NIR-images for a constant beam power ( $270 \mathrm{~W})$. Images from Fig. $\mathbf{2}$ are shown again to have a direct comparison between NIR-images and top surface images of the final parts. 


\subsection{Geometrical distortions and uneven top surfaces}

For the $U$ sample (see Table 1), an energy excess input was applied by decreasing the scanning speed resulting in an uneven sample. An uneven top surface can be seen in the optical image showing the final top surface once the build is completed (Fig. 6.b). The optical image also shows that the center of the sample appears relatively flat whereas the regions near the corners suffer from significant surface variations. This is a consequence of a typical mechanism reported and investigated by Körner et al. [22] based on numerical simulations using a Lattice-Boltzmann framework. This mechanism is described as a material displacement phenomenon. Because of the low-pressure environment in combination with high beam energy, evaporation of some alloying elements from the melt pool takes place. Thus, the recoil pressure induced by the evaporated material causes this material relocation and gives an irregular printed surface. During the build of the next layers, the powder is spread unevenly on that surface, the same phenomenon occurs, amplifies the defect, and finally gives important surface variations.

Samples produced with an excessive energy input appear brighter (i.e. higher grey level) in the in-situ NIR-images (Fig. 6.b). This can be simply attributed to the higher surface temperature induced by this high energy input. It should also be noted that the order in which parts are melted affects the grey level. The surface variations described previously cannot be captured explicitly in the in-situ NIR-images. One could expect to see varying depths as observed in the final top surface optical image, however, as the $700-1000 \mathrm{~nm}$ band-pass filter helps to reduce the reflected light, we assume that the surface variations are not sharp enough to induce local emissivity changes which would be seen in such images [25].

Fig. 7 shows the case of the sample U1 (processing conditions given in Table 2) where good processing parameters were first applied (linear energy of $0.85 \mathrm{~J} / \mathrm{mm}$, for the lower part of the build, i.e. build height $<17.7 \mathrm{~mm}$ ) before a decrease of the scanning speed in the upper part of the build (build height $>17.7 \mathrm{~mm}$, linear energy of $1.72 \mathrm{~J} / \mathrm{mm}$ ) to obtain uneven melting conditions. NIR-images of four consecutive layers of this sample are shown respectively for good processing parameters (Fig. 7.a) and uneven melting conditions (Fig. 7.b). Boundaries of the printed parts are shown in red in Fig. 7.b and were determined automatically by the image 
processing routine described in 2.3. Boundaries of the theoretical CAD area are shown in green. While the boundaries of the part match relatively well the theoretical CAD ones for good melting conditions as shown in Fig. 7.a, a deviation is revealed in Fig. 7.b for uneven melting conditions where there is a clear difference between the theoretical CAD boundary and the printed one. Here, high beam energy leads to very large melt pools that overpass the expected boundaries. It can be seen on each image that there is a deviation of the printed boundary along the downstream edge of the sample when it is melted, see for example at the bottom of the image taken at a building height of $19.60 \mathrm{~mm}$ (Fig. 7.b). This can be attributed to the hatching direction which, for this given layer, is from top to bottom as indicated by the black arrow. This deviation from the CAD geometry is also clearly visible in the following layers with similar melting conditions, the only difference being the displacement of this particular region which is rotated by $90^{\circ}$ at every new layer because the melting strategy is set so that the hatching direction rotates $90^{\circ}$ between each layer.

Fig. 7.c shows the evolution of the relative printed area as a function of the building height of the sample U1, where 1 corresponds to the surface printed with good melting condition. Printed areas were determined based on the in-situ NIR-images as described in section 2.3. It can be seen that the energy variation applied from a height of $17.7 \mathrm{~mm}$ can be clearly detected. For suitable processing parameters, the area remains stable around 1. However, as soon as the linear energy is increased, this area immediately increases and reaches a plateau between 1.03 and 1.07 after a few layers. The measured average overflow length of the downstream edge is $0.8 \mathrm{~mm}$. Note that the error bars corresponds to the error made on the area (overestimation or underestimation of the area) if the boundary is shifted by 1 pixel on each side of the determined boundary during image processing of NIR-images. 

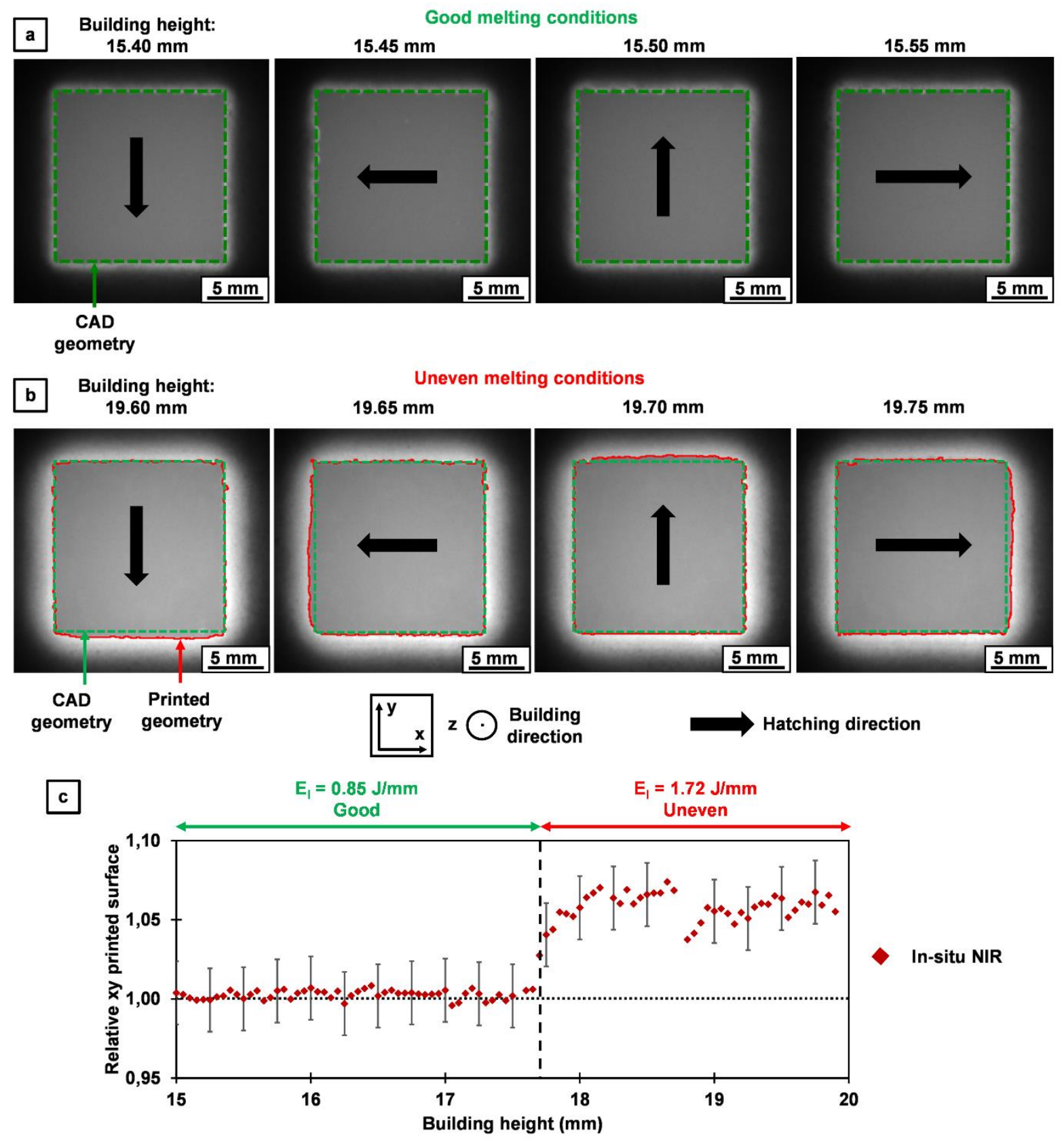

Fig. 7. (a) In-situ NIR-images of the sample U1 with good melting conditions taken for 4 consecutive layers from 15.4 $\mathrm{mm}$ to $15.55 \mathrm{~mm}$ (the final build height is $20 \mathrm{~mm}$ ) and (b) in-situ NIR-images of the same sample U1 but with uneven melting conditions taken for 4 consecutive layers from $19.6 \mathrm{~mm}$ to $19.75 \mathrm{~mm}$. Boundaries of the theoretical CAD area and the printed geometry are also emphasized for the sake of clarity. Each image corresponds to a different hatching direction; the latter being indicated by a black arrow. The difference between boundaries which is appearing for uneven melting conditions is clearly related to the hatching direction. (c) Evolution of the printed area based of the sample U1 on NIR-images (1 corresponds to the average printed area when using good melting conditions). An increase of this surface can be detected when a too high energy input is applied. 
Another typical defect is the geometrical distortion which can be observed near overhangs when supports are missing. For the sample G1 (see processing conditions in Table 2), the right part of the support structure was deliberately removed from the building file. Fig. 8.a shows an in-situ NIR-image showing the missing support taken at a building height of $8 \mathrm{~mm}(2 \mathrm{~mm}$ before the first layer of the cuboid sample). A second image of the same part has been taken at a building height of $11.15 \mathrm{~mm}$ (i.e. $1.15 \mathrm{~mm}$ above the first layer of the part, see Fig. 8.b). On the right side, this image looks brighter than in the rest of the part. Note that grey levels on images are linked to differences of temperature and differences of emissivity. The latter changes depending on the nature of the substrate: dense material vs. powder bed. Here, as the substrate is restricted to the melted part (no change of the nature of the substrate), it can be concluded that the temperature is locally higher near the region of the part where the support is missing because heat dissipation is here less efficient due to the difficulty to release heat through the powder bed. This can be critical when building overhang geometries because the powder underneath is not enough consolidated to efficiently release heat. Without supports, the overhang geometry is submitted to cyclic cooling and heating steps which induce thermal stresses that might lead to major distortions as previously shown in the literature $[21,26]$. This abnormal brightness tends to be reduced with the addition of new layers when looking far away from the overhang. The distortion of the final part is illustrated in Fig. 8.c.

No geometrical distortion is detected in Fig 8.b meaning that overhang distortions do not take place while melting, or at least do not appear as severe as it can be observed after examination of the final parts once the build is completed. It means that the qualitative relative thermal indications inferred from NIR-images can be a key piece of information for this kind of defect. Here, the flaw itself is not clearly visible, however, in-situ images give the operator a clue about the possible cause of this defect (insufficient heat dissipation). Such a signal in the NIR-images can be considered as an efficient way to suspect the formation of defects, and to identify the regions of a part that would require particular attention with post-processing controls or a CAD redesign. 


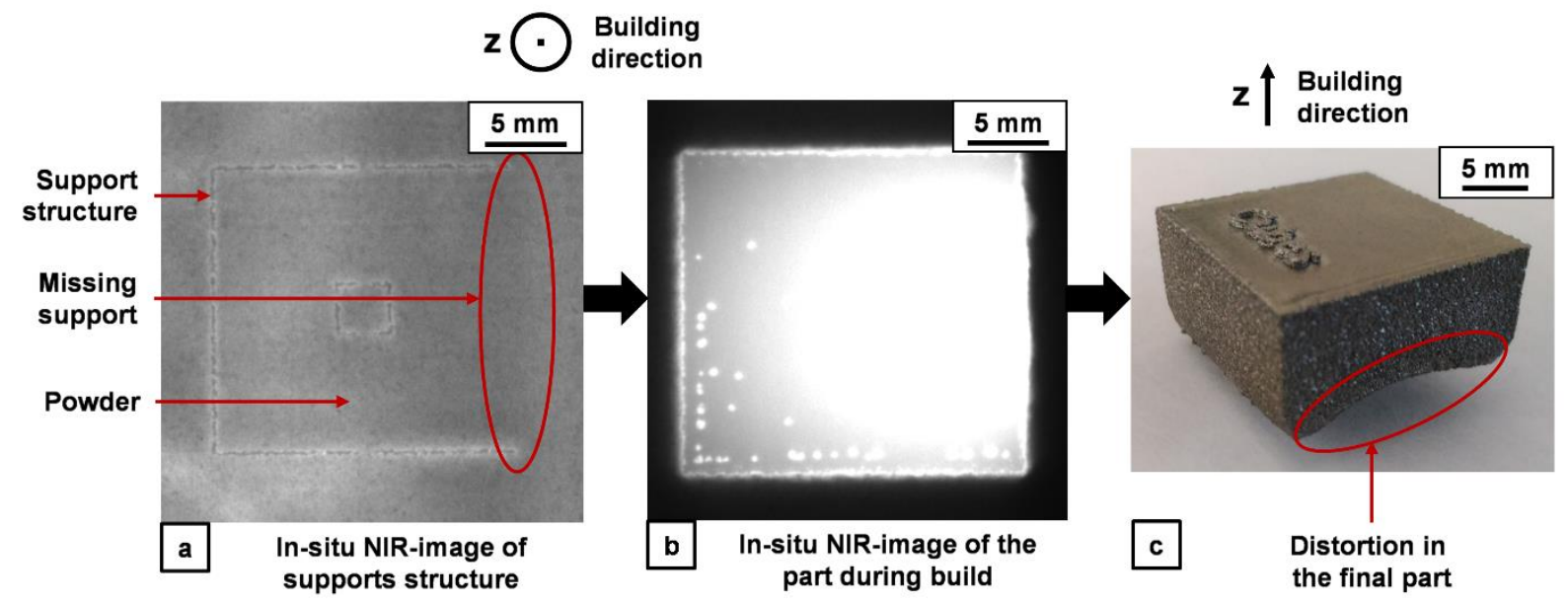

Fig. 8. (a) In-situ NIR-image of the support structure taken at a building height of $8 \mathrm{~mm}(2 \mathrm{~mm}$ underneath the first layer of the G1 sample). The support on the right is missing. (b) In-situ NIR-image of the G1 sample during the build taken at a building height of $11.15 \mathrm{~mm}$ (1.15 mm above the first layer of the part). High brightness can be seen on the right of the cube because of the missing support: heat is not similarly dissipated as in the case of the presence of a support. (c) Distortion observed in the final G1 sample due to the missing support.

\subsection{Porosity}

Some pores can be detected by NIR-imaging. A typical case is a situation where melting conditions lead to porous samples ( $\mathrm{P}$ in Table 1). Such processing conditions correspond to a lack of energy input, obtained here by increasing the scanning speed. Thus, the supplied energy is not sufficient to obtain nearly fully-dense materials (relative density $>99.8 \%$ ), therefore lack-of-fusion defects are generated. As illustrated in Fig. 6.d, a porous surface is observed in the optical image of the top surface once the build is completed. The NIR-images associated with this sample show a lot of spots that appear brighter in comparison with the rest of the part. These spots are pores that are acting as blackbody cavities $[11,16]$. In other words, it means that the emissivity is locally increased due to the presence of pores making them brighter. Note that pores appear brighter in the NIR-images but it does not necessarily mean that they are hot spots, i.e. regions subjected to temperature rises.

The sample P1 (processing conditions given in Table 2) was produced such that the lower part was built using good processing parameters (G-range), and then the processing parameters were 
deliberately changed from a given height to generate lack-of-fusion defects (P-range) in the upper part of the sample as illustrated in Fig. 9.a. Corresponding NIR-images are shown in Fig. 9.b (Grange) and Fig. 9.c (P-range).

3D images of a given region of interest extracted from this sample were obtained with, respectively, processed in-situ NIR-images (Fig. 9.d), and XCT-scans of the final part (Fig. 9.e). The comparison between 2D vertical cross-sections from NIR-images (Fig. 9.f) and XCT-scans (Fig. 9.g) is also given. Some small pores with a spherical morphology, which are colored in red, can be seen in the XCT 3D-image as well as in the XCT 2D cross-section but not in the NIR images. These pores are commonly observed in samples made by E-PBF and their characteristic round shape indicates that they are related to gas bubbles which were initially trapped in powder particles [1,27] during the atomization process. It is not possible to capture those kinds of pores in the NIR-images (even with a better spatial discretization) because those pores are always located underneath the top surface. We remind that only the top surface (surface under construction) is imaged with the NIRcamera during the build. On the contrary, most of the lack-of-fusion defects are connected to the surface under construction and are thus detected in the NIR-images. It is not possible to demonstrate that all the pores observed in NIR-images are surface connected. One could expect that a sub-surface pore would induce a different NIR signature because the heat transfer would be affected by the cavity and would be different from the surrounding dense material. However, we have never observed that.

Lack-of-fusion defects are detected in the upper part of the 3D images of the region of interest. In both 3D images, elongated pores with connections between them can be observed. Those pores are distributed rather homogeneously within the region of the part built with $\mathrm{P}$ melting conditions. Qualitatively, there is a good agreement between the in-situ characterization based on NIR-images acquired at every layer, and the post-processing XCT characterization, in particular for the shape and spatial distribution of pores. Pores in the 3D NIR-images seem coarser than the ones observed in the XCT 3D image. However, one should note that a quantitative comparison would not make sense because the spatial discretization of each imaging technique turns out to be too significantly different $\left(75 \times 75 \times 50 \mu \mathrm{m}^{3}\right.$ voxel size for NIR-images vs. $15 \times 15 \times 15 \mu \mathrm{m}^{3}$ voxel size in XCT images). 
The underlying mechanism of these large lack-of-fusion defects which are propagating along the building direction was rationalized by Bauereiß et al. [28] with the help of numerical simulations. They suggested that first, small irregularities on a given printed layer can act as initiation sites for such defects. For low energy inputs, these defects propagate along the building direction mainly because of the stochastic nature of the powder bed and of the predominance of surface tension in comparison with gravity. The surface tension tends to pull the melt pool out of the cavity and leads to defect growth as long as the beam energy is not sufficiently increased, see Bauereiß et al. [28] for more details. The latter observation partly explains why, to avoid the formation of such defects in E-PBF, the energy input is often selected to obtain melt pool depths which are at least 3 times the nominal layer thickness. The defect propagation mechanism and the fact that it cannot be corrected when processing next layers (if the beam energy is not increased) explains the good agreement between the 3D NIR-image and the XCT 3D image. Thus, when this defect is detected in NIR-images during the build, it is likely that it will still be present in the final part. The comparison between 2D vertical cross-sections obtained respectively by NIR-imaging (Fig. 9.f) and XCT (Fig. 9.g) demonstrates once again the relatively good agreement between in-situ and postprocessing characterizations. 


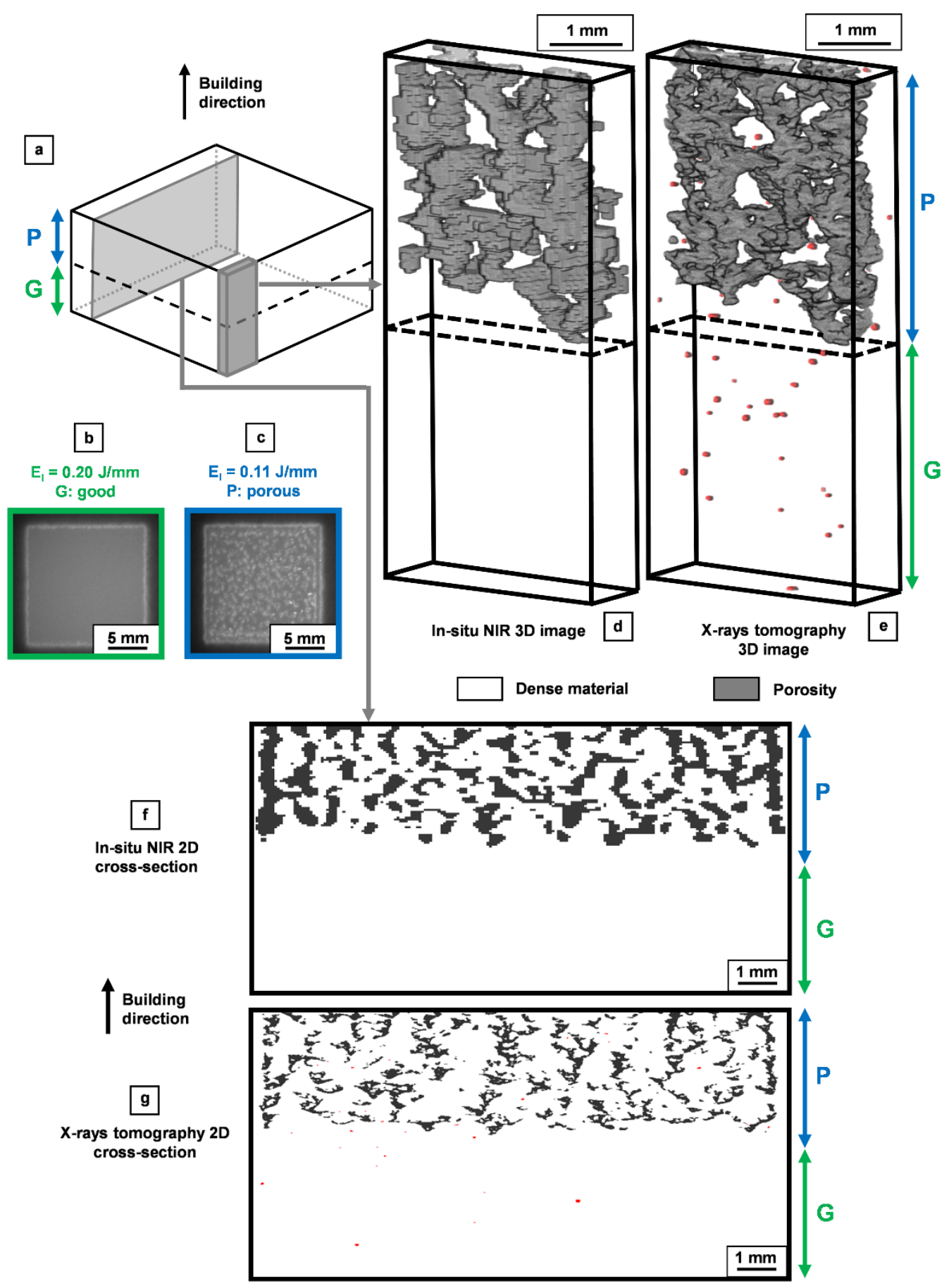

Fig. 9. Lack-of-fusion defects observed in the sample P1 (a). Suitable melting conditions were first applied in the lower part of the sample (b); linear energy was then decreased giving lack-of-fusion defects (c). A 3D in-situ NIR-image including a region with lack-of-fusion defects (d) is compared to a XCT 3D image of the same region of interest (e). Similarly, a 2D in-situ NIR vertical cross-section of the sample (f) is compared to the same cross-section obtained by XCT (g). The difference in spatial discretization between the two imaging techniques is clearly highlighted. Small rounded shape pores, visible only on XCT images are shown in red. 
In the previous case corresponding to deliberately insufficient energy input (sample P1), we have reported the occurrence of lack-of-fusion defects that were found to be rather homogeneously distributed within the part. However, we also found situations where such pores appear in a more isolated way. For instance, in sample G2 (processing conditions are given in Table 2) highly isolated pores were identified based on in-situ NIR-images. As already pointed out, those pores are not homogeneously distributed as seen in Fig. 10 showing a comparison between the previously described lack-of-fusion defects (Fig. 10.a) and these isolated pores (Fig 10.b). However, one should note that both types of pores have the same signature in NIR-images (bright spots) because of the same blackbody cavity effect. Here, it is worth noting that most of those isolated pores are located at the border between the contour and hatching regions. They show a particular morphology which is exemplified in Fig. 10.c and $\mathbf{d}$ where in-situ NIR and XCT 3D images of a similar region of interest are shown. These defects can be described as isolated elongated pores propagating along the building direction. Interestingly, those elongated pores seem to terminate quite randomly. There are no interconnections between them as seen for the lack-offusion defects described previously. Fig 10.e and $\mathbf{f}$ show an enlarged view of one of these pores which divides into two distinct branches. A relatively good agreement is found between in-situ NIR and XCT 3D images for the pores morphology as well as their spatial distribution within the sample. It is important to highlight that the camera can capture this kind of unexpected defects.

Such pores were defined as "chimney pores" by Cordero et al. [16]. Similar morphologies of pores were reported in the literature by other authors for samples produced by E-PBF $[28,29]$. The propagation mechanism seems to be identical than the one at the origin of the previously described lack-of-fusion defects, i.e. linked with low energy inputs. However, in our case, these isolated elongated defects are observed though they are not induced by deliberately modified beam parameters. Standard ARCAM processing parameters were employed for this sample. A possible explanation of this defect growth could be accounted for a non-optimized beam calibration. In such a case, the beam would be defocused and therefore would decrease the energy density allowing chimney pore to be grown. This assumption is also consistent with the fact that all the parts for which those chimney pores were found, were all manufactured in the same build. However, this assumption does not necessarily explain why the interface between 
contour and hatching regions seems to be a preferential zone for these pores. A similar issue was reported previously in the literature by Tammas-Williams et al. [29] where some chimney pores were found at the edges of the hatching zone. This was attributed to a locally lower energy input because the ARCAM software increases the beam scanning speed when leaving the edges of the hatching zone (turning point function). In our case, we cannot exclude the same issue reported in [29] by Tammas-Willimas et al., but in such a case we would expect to observe those defects along the edges of the hatching zone, however, the chimney pores detected were not found to be only distributed along all the edges but rather localized here and there. Further investigation would be required to rationalize the underlying mechanism responsible for defect initiation and termination. This has been considered out of the scope of the present study whose main objective is rather to demonstrate that NIR-images can be an efficient way for layerwise monitoring of EPBF. 

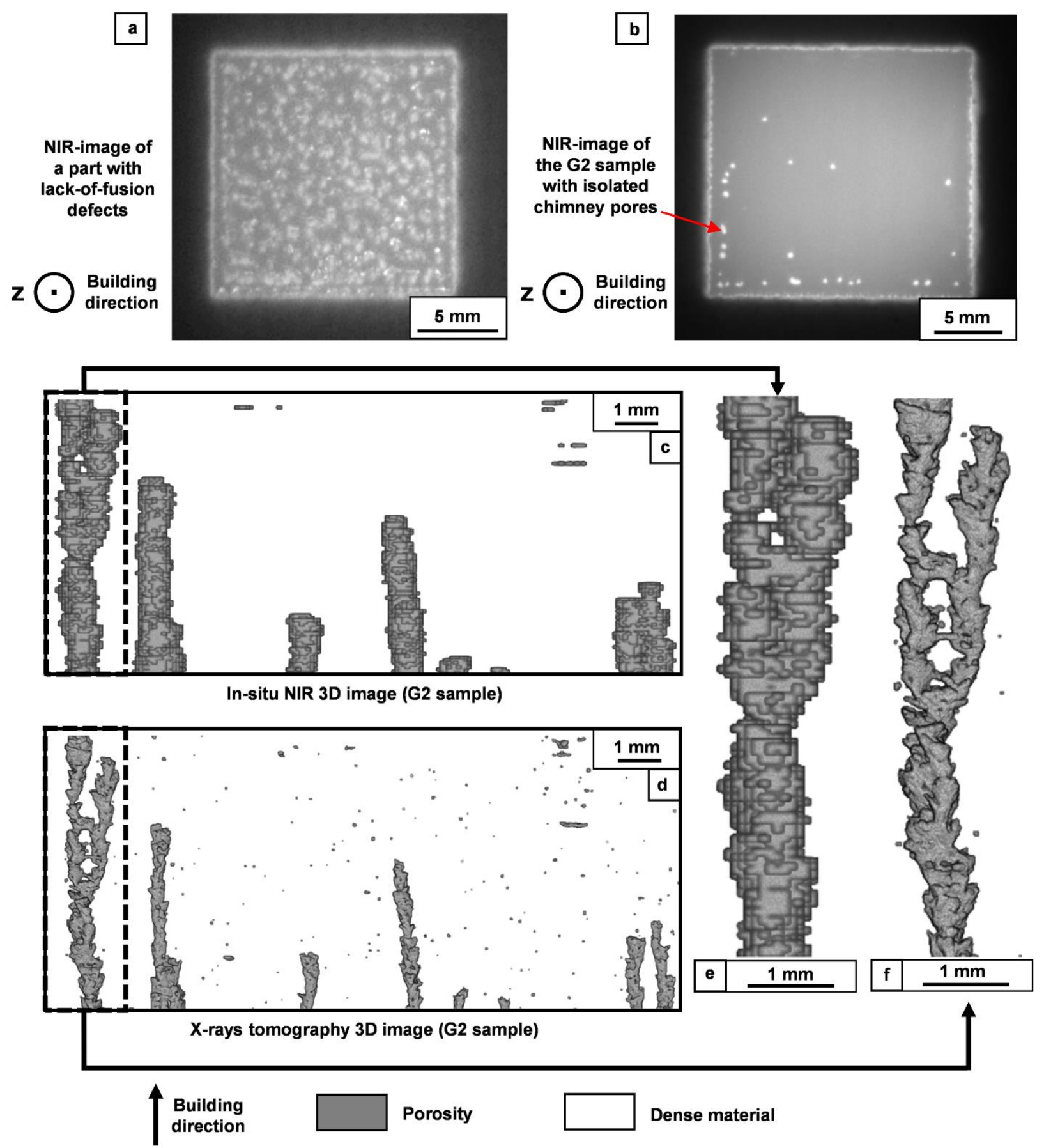

Fig. 10. Comparison between NIR-images of lack-of-fusion defects (a) and chimney pores observed in the sample G2 (b). 3D rendering of the same region of interest in the G2 sample showing chimney pores as revealed by (c) in-situ NIR-images and (d) XCT scans. 3D rendering of the same chimney defect from (e) in-situ NIR-images with a 75x75x50 $\mu \mathrm{m}^{3}$ voxel size and (f) XCT scans with a $15 \times 15 \times 15 \mu \mathrm{m}^{3}$ voxel size. 


\section{Conclusion}

A high spatial discretization NIR camera is implemented in an E-PBF ARCAM A1 machine. For each built layer, an image is taken making it possible to image typical defects as well as to provide qualitative thermal information. This work allows to validate that typical E-PBF defects could be effectively detected with NIR-imaging as reported in the literature. In-house image analysis routines allow the in-situ detection of these defects to be substantially improved. Detection of defects in NIR-images allows the processing window for a given material to be determined more efficiently.

Uneven samples induced by an excess of energy input are revealed by in-situ NIR-images by a geometric deviation in comparison with the expected CAD dimensions. Distortions occurring near overhangs are also qualitatively identified thanks to the thermal information provided by NIRimages. Lack-of-fusion defects captured in-situ through 3D NIR-images are compared to the defects characterized in final parts by XCT. Qualitatively, a good agreement is found for the defect morphology and position making in-situ NIR images a reliable method to detect defects during a build. Chimney pores are also detected and a good agreement is observed once again between 3D images from in-situ NIR-images on the one hand, and XCT on the other hand. The results of the present work should enable better control of the E-PBF process, and ultimately open the path towards a closed-loop control that would help to make those processing routes more reliable.

Additional work would be needed to further improve image analysis routines in order to have a completely automatic defect detection with immediate image processing and standardized acquisition conditions during E-PBF builds. Materials exhibiting drastically different optical or physical properties in comparison with Ti-alloys could be interesting to investigate using in-situ NIR-images to evaluate the consequences on defect detection. In particular, materials with very low emissivity/high reflectivity such as copper or aluminum alloys would be good candidates. 


\section{Acknowledgments}

The authors gratefully acknowledge the FUI PALOMA research program for funding and fruitful discussions with Ludovic Ropars, leader of this research project. This work was supported by the Centre of Excellence of Multifunctional Architectured Materials "CEMAM" n ${ }^{\circ}$ N-10-LABX-44-01 funded by the "Investments for the Future" program. The authors are respectively thankful to Alain Di Donato and Julie Maisonneuve for their help and support for the implementation of the camera in the E-PBF machine.

\section{References}

[1] S. Tammas-Williams, H. Zhao, F. Léonard, F. Derguti, I. Todd, P.B. Prangnell, XCT analysis of the influence of melt strategies on defect population in Ti-6Al-4V components manufactured by Selective Electron Beam Melting, Mater. Charact. 102 (2015) 47-61. https://doi.org/10.1016/j.matchar.2015.02.008.

[2] T. Persenot, G. Martin, R. Dendievel, J.-Y. Buffiére, E. Maire, Enhancing the tensile properties of EBM as-built thin parts: Effect of HIP and chemical etching, Mater. Charact. 143 (2018) 82-93. https://doi.org/10.1016/j.matchar.2018.01.035.

[3] V. Juechter, T. Scharowsky, R.F. Singer, C. Körner, Processing window and evaporation phenomena for Ti-6Al-4V produced by selective electron beam melting, Acta Mater. 76 (2014) 252-258. https://doi.org/10.1016/j.actamat.2014.05.037.

[4] S.K. Everton, M. Hirsch, P. Stravroulakis, R.K. Leach, A.T. Clare, Review of in-situ process monitoring and in-situ metrology for metal additive manufacturing, Mater. Des. 95 (2016) 431445. https://doi.org/10.1016/j.matdes.2016.01.099.

[5] M. Mani, B.M. Lane, M.A. Donmez, S.C. Feng, S.P. Moylan, A review on measurement science needs for real-time control of additive manufacturing metal powder bed fusion processes, Int. J. Prod. Res. 55 (2017) 1400-1418. https://doi.org/10.1080/00207543.2016.1223378.

[6] M. Grasso, B.M. Colosimo, Process defects and in situ monitoring methods in metal powder bed fusion: a review, Meas. Sci. Technol. 28 (2017) 044005. https://doi.org/10.1088/13616501/aa5c4f.

[7] C. Arnold, C. Pobel, F. Osmanlic, C. Körner, Layerwise monitoring of electron beam melting via backscatter electron detection, Rapid Prototyp. J. 24 (2018) 1401-1406. https://doi.org/10.1108/RPJ-02-2018-0034.

[8] H. Wong, D. Neary, S. Shahzad, E. Jones, P. Fox, C. Sutcliffe, Pilot investigation of feedback electronic image generation in electron beam melting and its potential for in-process monitoring, J. Mater. Process. Technol. 266 (2019) 502-517. https://doi.org/10.1016/j.jmatprotec.2018.10.016. 
[9] C.R. Pobel, C. Arnold, F. Osmanlic, Z. Fu, C. Körner, Immediate development of processing windows for selective electron beam melting using layerwise monitoring via backscattered electron detection, Mater. Lett. 249 (2019) 70-72. https://doi.org/10.1016/j.matlet.2019.03.048.

[10] S. Price, J. Lydon, K. Cooper, K. Chou, Experimental temperature analysis of powder-based electron beam additive manufacturing, in: 24th Annu. Int. Solid Free. Fabr. Symp. Austin, TX, 2013: pp. 162-173.

[11] N. Boone, C. Zhu, C. Smith, I. Todd, J.R. Willmott, Thermal near infrared monitoring system for electron beam melting with emissivity tracking, Addit. Manuf. 22 (2018) 601-605. https://doi.org/10.1016/j.addma.2018.06.004.

[12] M. Grasso, G. Valsecchi, B.M. Colosimo, Powder bed irregularity and hot-spot detection in electron beam melting by means of in-situ video imaging, Manuf. Lett. 24 (2020) 47-51. https://doi.org/10.1016/j.mfglet.2020.03.011.

[13] S. Yoder, S. Morgan, C. Kinzy, E. Barnes, M. Kirka, V. Paquit, P. Nandwana, A. Plotkowski, R.R. Dehoff, S.S. Babu, Characterization of topology optimized Ti-6Al-4V components using electron beam powder bed fusion, Addit. Manuf. 19 (2018) 184-196. https://doi.org/10.1016/j.addma.2017.12.001.

[14] J. Schwerdtfeger, R.F. Singer, C. Körner, In situ flaw detection by IR-imaging during electron beam melting, Rapid Prototyp. J. 18 (2012) 259-263. https://doi.org/10.1108/13552541211231572.

[15] R.B. Dinwiddie, R.R. Dehoff, P.D. Lloyd, L.E. Lowe, J.B. Ulrich, Thermographic in-situ process monitoring of the electron-beam melting technology used in additive manufacturing, in: Proc. SPIE 8705, Thermosense Therm. Infrared Appl. XXXV, 2013: p. 87050K. https://doi.org/10.1117/12.2018412.

[16] Z.C. Cordero, R.B. Dinwiddie, D. Immel, R.R. Dehoff, Nucleation and growth of chimney pores during electron-beam additive manufacturing, J. Mater. Sci. 52 (2017) 3429-3435. https://doi.org/10.1007/s10853-016-0631-z.

[17] E. Rodriguez, J. Mireles, C.A. Terrazas, D. Espalin, M.A. Perez, R.B. Wicker, Approximation of absolute surface temperature measurements of powder bed fusion additive manufacturing technology using in situ infrared thermography, Addit. Manuf. 5 (2015) 31-39. https://doi.org/10.1016/j.addma.2014.12.001.

[18] J. Raplee, A. Plotkowski, M.M. Kirka, R. Dinwiddie, A. Okello, R.R. Dehoff, S.S. Babu, Thermographic Microstructure Monitoring in Electron Beam Additive Manufacturing, Sci. Rep. 7 (2017) 43554. https://doi.org/10.1038/srep43554.

[19] R.B. Dinwiddie, M.M. Kirka, P.D. Lloyd, R.R. Dehoff, L.E. Lowe, G.S. Marlow, Calibrating IR cameras for in-situ temperature measurement during the electron beam melt processing of Inconel 718 and Ti-Al6-V4, in: Proc. SPIE 9861, Thermosense Therm. Infrared Appl. XXXVIII, 2016: p. 986107. https://doi.org/10.1117/12.2229070.

[20] S.S. Sih, J.W. Barlow, The Prediction of the Emissivity and Thermal Conductivity of Powder Beds, Part. Sci. Technol. 22 (2004) 427-440. https://doi.org/10.1080/02726350490501682.

[21] B. Cheng, K. Chou, Geometric consideration of support structures in part overhang fabrications by electron beam additive manufacturing, Comput. Des. 69 (2015) 102-111. https://doi.org/10.1016/j.cad.2015.06.007. 
[22] C. Körner, Additive manufacturing of metallic components by selective electron beam melting a review, Int. Mater. Rev. 61 (2016) 361-377. https://doi.org/10.1080/09506608.2016.1176289.

[23] C.O.S. Sorzano, P. Thévenaz, M. Unser, Elastic registration of biological images using vector-spline regularization, IEEE Trans. Biomed. Eng. 52 (2005) 652-663.

https://doi.org/10.1109/TBME.2005.844030.

[24] I. Arganda-Carreras, C.O.S. Sorzano, R. Marabini, J.M. Carazo, C. Ortiz-de-Solorzano, J. Kybic, Consistent and Elastic Registration of Histological Sections Using Vector-Spline Regularization, in: Comput. Vis. Approaches to Med. Image Anal. CVAMIA 2006. Lect. Notes Comput. Sci. Vol 4241, 2006: pp. 85-95. https://doi.org/10.1007/11889762_8.

[25] T.-Y. Cheng, D. Deng, C. Herman, Curvature Effect Quantification for In-Vivo IR Thermography, in: Proc. ASME 2012 Int. Mech. Eng. Congr. Expo. Vol. 2 Biomed. Biotechnol., 2012: pp. 127-133. https://doi.org/10.1115/IMECE2012-88105.

[26] T.H. Vo, M. Museau, F. Vignat, F. Villeneuve, Y. Ledoux, A. Ballu, Typology of geometrical defects in Electron Beam Melting, Procedia CIRP. 75 (2018) 92-97. https://doi.org/10.1016/j.procir.2018.04.033.

[27] X. Gong, T. Anderson, K. Chou, Review on Powder-Based Electron Beam Additive Manufacturing Technology, in: Proc. ASME/ISCIE 2012 Int. Symp. Flex. Autom., 2012: pp. 507-515. https://doi.org/10.1115/ISFA2012-7256.

[28] A. Bauereiß, T. Scharowsky, C. Körner, Defect generation and propagation mechanism during additive manufacturing by selective beam melting, J. Mater. Process. Technol. 214 (2014) 25222528. https://doi.org/10.1016/j.jmatprotec.2014.05.002.

[29] S. Tammas-Williams, P.J. Withers, I. Todd, P.B. Prangnell, The Effectiveness of Hot Isostatic Pressing for Closing Porosity in Titanium Parts Manufactured by Selective Electron Beam Melting, Metall. Mater. Trans. A. 47 (2016) 1939-1946. https://doi.org/10.1007/s11661-016-3429-3. 